\title{
Research on the Relationship Between Servant Leadership and Employee Innovation Behavior: A Theoretical Model
}

\author{
Shiwen $\mathrm{Liao}^{1,{ }^{*}}$ and $\mathrm{Xin} \mathrm{Zhu}^{1,2}$ \\ ${ }^{1}$ School of Economics and Management, Guangxi University of Science and Technology, Liuzhou, Guangxi 545006, \\ China \\ ${ }^{2}$ School of Management, Huazhong University of Science and Technology, Wuhan, Hubei 430074, China \\ *Corresponding author. Email: 623273036@qq.com
}

\begin{abstract}
As a leadership style characterized by "altruism" and "service", servant leadership is a frontier topic in the field of leadership research in recent years. Based on social exchange theory and social cognitive theory, this paper constructs a model of the impact of servant leadership on employees' innovation behavior, and takes employees' perceived insider status as a mediating variable to explain the internal interaction mechanism between them, which lays a foundation for empirical research. This research is beneficial to further expand the research results of the theory of innovation behavior, and provide theoretical and practical guidance for enterprise innovation management.
\end{abstract}

Keywords: servant leadership, employee innovation behavior, perceived insider status

\section{INTRODUCTION}

With increasingly fierce global competition, rapid technological revolution, innovation has gradually become the internal driving force for the survival and development of contemporary organizations. As the source and starting point of organizational innovation, the effective stimulation of employees' innovative behavior has become an important source for organizations to gain competitive advantage. Therefore, how to stimulate employees' enthusiasm for innovation has become an urgent problem to be solved, and the servant leadership theory provides a new perspective for organizational practice and scholars' research. Contrary to the traditional power-driven, selfservice and leadership-first paradigm, servant leadership emphasizes "service-first", takes employees as the center, puts their needs and interests in the first place, and pays attention to the growth and development of employees, which can promote the positive behavior of employees.

However, the influence of leadership style on employees' behavior is often indirect, which is transmitted through employees' inner psychology and cognition. At present, China's economy is in the period of transition. Layoffs, dismissals and re-sign labor contracts have become problems that employees worry about and may occur at any time. In this environment, an important problem faced by organizations is how to make employees have a sense of psychological security and a sense of belonging to the organization, so as to stimulate the enthusiasm and creativity of employees and improve the performance of the organization. Perceived insider status is an important variable to explain the relationship between employee and organization, which reflects the quality of employment relationship, and its importance has been recognized by scholars at home and abroad, it is regarded as an important condition to stimulate employee's positive behavior. Therefore, how to improve the employee's perceived insider status is a key problem for managers. Solving this problem is of great significance to the management practice of Chinese organizations under the current situation.

\section{CONCEPT AND CONNOTATION}

\subsection{Servant Leadership}

Greenleaf believes that servant leadership is a leadership style that transcends the personal interests of leaders, emphasizes "serving others" and puts the needs, wishes and interests of employees at the top priority[1]. Different from other leadership styles, servant leaders are service-oriented and altruistic managers, rather than showing their own unique status and identity.

\subsection{Perceived Insider Status}

Stamper and Masterson put forward the concept of perceived insider status according to the way subordinates feel being treated differently in the organization, it refers to the value that subordinates feel about themselves and the degree to which they are treated as "insiders" by the organization, reflects the cognition of subordinates on their 
employment relationship[2]. According to the characteristics of collectivism in Chinese organizational culture, $\mathrm{Yi}$ et al. define perceived insider status as the extent to which subordinates perceive themselves as "members of a group" or "masters" in an organization, emphasizing "sense of belonging" and "sense of integration".

\subsection{Employee Innovative Behavior}

Zhou and Georg hold that personal innovation is the starting point of organizational innovation, and employees' innovation behavior is not only the generation of innovative ideas, but also the promotion and implementation of innovative ideas, so as to ensure the effective implementation of innovative ideas[3]. Yuan and Woodman believe that employees' innovation behavior is a complex behavior combination of generating ideas, introducing ideas, implementing ideas.

\section{THEORETICAL BASIS}

\subsection{Social Exchange Theory}

Homans puts forward social exchange theory based on the individual level, holding that the essence of interpersonal communication in society is an exchange relationship, which aims to explain the process of social behavior such as leadership, power, status, fairness and justice. Gouldner puts forward the principle of reciprocity based on the social exchange theory, which can solve the problems caused by the inconsistent time of the two sides' behaviors in the exchange process, effectively reduce the exchange risk, and help the exchange parties to achieve mutual benefit. Blau applies social exchange theory based on the organizational level, he holds that the two sides of social exchange provide valuable things to each other, and the beneficiary will try every means to repay the favor until the exchange is balanced, if only one party provides resources and rewards, but the other does not pay due response, because of the lack of the principle of equality and reciprocity, exchange activities will not happen[4].

\subsection{Social Cognitive Theory}

Bandura puts forward social cognitive theory, which holds that environment, individual cognition and behavior constitute a constant dynamic and interactive triangular relationship. Bandura specifically explore the interaction among the three elements concerned in social cognitive theory: First, individual's self-confidence, emotion, belief and other cognitive factors affect the individual's behavior, similarly, the results of behavior are fed back to the individual's cognition. Secondly, environment, as the external condition of behavior, determines the direction and intensity of behavior, and behavior can change the environment to meet individual needs. Finally, there is interaction between environment and cognition, individual cognition is formed in the objective environment and is adjusted by the environment, while individual thinking and cognitive style can form group changes through communication, and then affect the environment[5]. In organizational management, the behavior of subordinates is a process of interaction among organization-related factors, subordinates' cognition and behavior. Organizational situational factors such as human resource management practices, leadership style and organizational culture affect subordinates' work attitude, work motivation and cognition, and then affect their behavior, work result and performance.

\section{THEORETICAL MODEL}

\subsection{Servant Leadership and Employee Innovation Behavior}

As a leadership style that transcends personal interests, servant leaders care about employees' work and life, attach importance to employees' career development, encourage employees to make their own decisions, and help employees solve work problems, fully meet employees' material and spiritual needs[6]. These service-oriented leadership behaviors can be regarded as a leader's resource investment to establish good social exchange relationship with employees[7]. According to social exchange theory and the principle of reciprocity[4], when employees perceive the importance and recognition of leaders, they will consider that they have the obligation to repay good treatment and seek appropriate ways to repay them. One of the ways for employees to repay leaders is to implement extra-role behavior. As an innovation-oriented extra-role behavior, innovation behavior can provide innovative ideas and gain competitive advantages for the organization. Employees are willing to improve work efficiency and process and take certain risks to make innovation behavior. Bandura considers that the environment is the basis of behavior, and has certain guiding effect on behavior[5] The leader's behavior style and personality traits can be considered as an environment in the organization where the subordinates belong, subordinates construct their behaviors by obtaining information from the outside to be consistent with the external environment. Servant leaders focus on the personal development of their subordinates, cultivate their work skills and provide emotional support and material help, constantly promote the improvement of their skills and resources, thus creating a well-resourced environment[6]. According to social cognitive theory, in order to achieve the matching of environment and behavior, the subordinates show innovative behaviors to help the organization improve operating efficiency. 
Van Dierendonck and Patterson argue that servant leaders actively empower their employees and tolerate their mistakes[8]. On the one hand, the generation of employees' innovation behavior is a process including the generation, promotion and implementation of creativity, only when employees have mastered the autonomy can they promote innovation from idea to reality. The servant leader pays attention to empowerment, encourages employees to make their own decisions, and has created the necessary conditions for subordinates to participate in innovation. On the other hand, employees' innovative behavior is a process full of challenges and risks, which will inevitably meet with setbacks and failures. When innovation is frustrated, servant leaders will actively encourage and comfort the employees, even if employees fail in innovation, they will not be blamed by the leaders, which to some extent eliminate the worries of employees' innovation failure.

Therefore, this paper holds that servant leadership has a positive effect on employee innovation behaviour.

\subsection{Servant Leadership and Perceived Insider Status}

Schaubroeck et al. points out that leadership style can influence subordinates' perceived insider status, and positive leadership style can improve subordinates' perceived insider status[9]. On the one hand, servant leaders put subordinates in the first place, fully respect their dignity, affirm their value, take serving others as the first priority, and strive to meet the physiological, psychological and emotional needs of subordinates. According to social exchange theory, the information of care, respect, encouragement and recognition conveyed by servant leaders to their subordinates can make them feel that the relationship with the organization has surpassed the economic interests, and more importantly, it is a kind of social exchange relationship, which enables the subordinates to realize their importance to the organization, and then establish a long-term organizational commitment, regard the organization as their own home, and strengthen perceived insider status. On the other hand, servant leaders delegate appropriate authority to their subordinates in their work, signaling that the organization is investing in subordinates, and letting subordinates feel that they are trusted, valued, and nurtured by the organization. According to Stamper and Masterson, when subordinates perceive that their contributions to the organization are valued, they will consider themselves as a member of the organization[2], thus the level of subordinates' perceived insider status will be enhanced.

Therefore, this paper holds that servant leadership has a positive effect on perceived insider status.

\subsection{Perceived Insider Status and Employee Innovation Behavior}

In the context of the collectivism culture, employees' access to personal space and membership from the organization is the premise of making contributions to the organization, and individual definition of their role in the organization will affect their behavior[10]. Employees with high level of perceived insider status feel the importance and support of the organization, define themselves as part of the organization, meet their needs of emotional connection and sense of belonging[11]. According to social exchange theory and the principle of reciprocity, in order to reward the organization, employees will not only try their best to complete the in-role behavior, but also be willing to take the initiative to implement the work beyond the requirements of their role, breaking the routine, more novel and practical ideas will be put forward and applied to work, and innovative behavior will be effectively promoted.

Wang et al. point out that when employees feel that they are insiders of the organization, they will regard themselves as citizens of the organization and take on their due responsibilities, so as to actively carry out some activities beyond the scope of general job responsibilities[12]. Because employees with high level of perceived insider status are aware of the organization's acceptance and recognition of them, according to social cognitive theory, individual cognition is the basis of behavior, the employees with higher perceived insider status consider that the interests of the organization are closely related to their own interests, they should take more responsibility of the organization, and they are willing to devote more time and energy to the development of the organization, which stimulates the innovative thinking and innovative behavior of employees.

Therefore, this paper holds that perceived insider status has a positive effect on employee innovation behavior.

\subsection{The Mediating Role of Perceived Insider Status}

Based on CAPS, the external situation factors can trigger internal cognition and emotion and ultimately affect the individual behavior[13]. The service and altruistic behavior of servant leaders shows their concern, help and empowerment to employees, create a service-oriented management environment, which can convey the signals that they are part of the organization, employees will generate positive emotions and cognition, and then judge their self-identity, classify themselves as "insiders" and form a high-level perceived insider status[10]. Employees with a high level of perceived insider status can feel the trust and approval of the organization. According to Social exchange theory and the principle of reciprocity, employees will have the obligation to return, consciously undertake the work tasks beyond the responsibilities stipulated by the organization, accept the challenging work, actively explore new methods to solve problems, be brave 
in innovation, and promote the generation of employees' innovative behavior.

Therefore, this paper holds that perceived insider status plays mediating role between servant leadership and employee innovation behavior.

To sum up, the way leaders treat subordinates will affect their cognition of their status in the organization, and then affect their work attitude and behavior[14]. Through caring, empowering and serving, servant leaders make subordinates respected and get a sense of belonging, which makes subordinates have a high level of internal identity perception, the sense of belonging can encourage subordinates to explore and innovate, take the risk of innovation and engage in innovative behavior. Therefore, perceived insider status transmits the influence of servant leadership on employees' innovation behavior. The theoretical model of this paper is shown in Fig. 1.

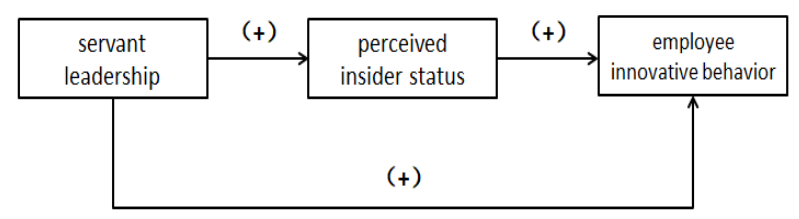

Figure 1. Theoretical Model

\section{THEORETICAL AND PRACTICAL IMPLICATIONS}

\subsection{Theoretical Implications}

First, it deepens the understanding of the mechanism of servant leadership and promotes the construction of the theoretical system of servant leadership. Most of the existing studies explore the influence of leadership style on employee behavior from a single perspective. Based on social exchange theory and social cognitive theory, this paper explores the relationship between variables from a multi-dimensional perspective and expands the theoretical interpretation perspective. On the one hand, from the perspective of social cognitive theory, this paper analyzes that servant leadership has laid a psychological foundation for employees' innovative behavior on the basis of improving employees' perceived insider status; On the other hand, based on the perspective of social exchange theory, it analyzes employees' perceived investment in employee development, which makes employees have the obligation to repay the organization through innovative behavior.

Secondly, it enriches the impact of servant leadership. Although servant leadership originated in the 1970s, the research on servant leadership in China is still in its infancy and is not mature enough. Based on China's organizational management situation, combined with the background of gradual narrowing of power distance and economic and social transformation, this paper discusses the impact of servant leadership on employees' psychological perception and behavior, reveals the positive influence of servant leadership on employees' innovation behavior, confirms the effectiveness of servant leadership to some extent, and expands the related research on the mediating variables and outcome variables of servant leadership.

Thirdly, it enriches the antecedent and outcome variables of perceived insider status. Taking perceived insider status as a mediating variable, it builds a bridge and a link between the servant leadership and the employee innovative behavior, discusses the role of employee's "sense of belonging" as an individual's positive perception of the organization in stimulating employee's innovative behavior, and forms a mechanism model of "SL $\rightarrow \mathrm{PIS} \rightarrow$ EIB", which further opens the "black box" of the relationship between servant leadership and employee innovation behavior, and reveals the internal mechanism.

\subsection{Practical Implications}

First, servant leadership has an important impact on employees' psychological perception and behavior. Leaders should not rely too much on formal power and rules to manage employees, and should pay more attention to the transformation of servant leadership style. On the one hand, when selecting leaders, organizations should pay more attention to whether the leaders possess better qualities of "serving others", and actively carry out the training of leaders' service skills and pay attention to cultivating the leaders' service consciousness, improve the service skills and management level of leaders. On the other hand, leaders should give full recognition and trust to their employees, fully respect their values, put their interests in the first place, actively authorize, create a trusting and positive organizational atmosphere, and stimulate employees' innovative behavior.

Secondly, perceived insider status is the bridge between servant leadership and employee innovation behavior. This means that leaders should take a variety of measures to constantly enhance employees' sense of belonging to the organization, stimulate employees' sense of ownership, and improve the level of perceived insider status, so as to promote them to participate in innovation activities more actively. On the one hand, the organization should establish fair, open and reasonable rules and regulations from the system, culture and other software construction, and create a cultural atmosphere of people-oriented, error tolerance and innovation encouragement. On the other hand, leaders should give full play to the positive role of servant leadership, provide learning opportunities for employees, pay attention to the career development of employees, and properly authorize to encourage employees to make decisions independently, so that employees can feel their own value in their work and feel the importance and recognition of the organization to them, so as to improve perceived insider status of employees and stimulate their innovation motivation. 


\section{CONCLUSION}

In the era of knowledge economy, without innovation, enterprises will lose the motive power of advancing continuously, how to stimulate employees' innovative ideas and promote their innovative behavior to the greatest extent has become the key to the survival and development of enterprises. Servant leadership is an important source for employees to obtain work resources and psychological resources, which has a positive impact on employees' cognition and behavior. This paper points out that improving the service level and quality of the leaders and strengthening the employees' perceived insider status have an important role in promoting the employees' innovation behavior, which provides a useful reference for enterprise reform and practice management.

\section{ACKNOWLEDGMENT}

This work was supported by Innovation Project of Guangxi University of Science and Technology Graduate education (GKYC202017).

\section{REFERENCES}

[1] Greenleaf, R. K. The Servant as a Leader[M]. Indianapolis, In: Greenleaf Center, 1970.

[2] Stamper, C. L., Masterson, S. S. Insider or outsider? how employee perceptions of insider status affect their work behavior[J]. Journal of Organizational Behavior, 2002, 23(8):pp.875-894

[3] Zhou, J., George, J. M. When Job Dissatisfaction Leads to Creativity: Encouraging the Expression of Voice[J]. Academy of Management Journal, 2001, 44(4):pp.682-696.

[4] Blau, P. M. Exchange and Power in Social Life[M]. New York: John Wiley, 1964.

[5] Bandura, A. Social Cognitive Theory: An Agentic Perspective[J]. Asian Journal of Social Psychology, 1999, 2(1):pp.21-41.

[6] Eva, N., Robin, M., Sendjaya, S., Van Dierendonck, D., Liden, R. C. Servant Leadership: A systematic review and call for future research $[\mathrm{J}]$. The Leadership Quarterly, 2019, 30(1):pp.111-132.

[7] Walumbwa, F. O., Hartnell, C. A., Oke, A. Servant Leadership, Procedural Justice Climate, Service Climate, Employee Attitudes, and Organizational Citizenship Behavior: A Cross-Level Investigation[J]. Journal of Applied Psychology, 2010, 95(3):pp.517-529.

[8] Van Dierendonck. D., Patterson, K. Compassionate Love as a Cornerstone of Servant Leadership: An Integration of Previous Theorizing and Research[J]. Journal of Business Ethics, 2015, 128(1):pp.119-131.

[9] Schaubroeck, J. M., Shen, Y., Chong, S. A DualStage Moderated Mediation Model Linking Authoritarian Leadership to Follower Outcomes[J]. Journal of Applied Psychology, 2017,102(2), pp. 213214.

[10] Wang, J., Kim, T. Y. Proactive socialization behavior in China: The mediating role of perceived insider status and the moderating role of supervisors' traditionality $[\mathrm{J}]$. Journal of Organizational Behavior, 2013, 34(3):pp.389-406.

[11] Knapp, J. R., Smith, B. R., Sprinkle, T. A. Clarifying the Relational Ties of Organizational Belonging[J]. Journal of Leadership \& Organizational Studies, 2014, 21(3):pp.273-285.

[12] Wang, Lin., Chu, Xiaoping., Ni, Jing. The Role Exchange between the Leader and the Subordinate, the cognition of insiders' status and the Behavior of Organizational Citizens[J]. Management world, 2009(01):pp.97-107+188.

[13] Mischel, W., Shoda, Y. A Cognitive-Affective System Theory of Personality: Reconceptualizing Situations, Dispositions, Dynamics, and Invariance in Personality Structure[J]. Psychological Review, 1995, 102 (2): pp.246-268.

[14] Tyler, T. R., Lind, E. A. A Relational Model of Authority in Groups[J]. Advances in Experimental Social Psychology, 1992, 25: pp.115-191. 